Chapman University

Chapman University Digital Commons

Pharmacy Faculty Articles and Research

School of Pharmacy

7-28-2016

\title{
Incorporating Indications into Medication Ordering — Time to Enter the Age of Reason
}

Gordon Schiff

Brigham and Women's Hospital

Enrique Seoane-Vazquez

Chapman University, seoanevazquez@chapman.edu

Adam Wright

Brigham and Women's Hospital

Follow this and additional works at: http://digitalcommons.chapman.edu/pharmacy_articles

Part of the Health and Medical Administration Commons, and the Pharmacy Administration, Policy and Regulation Commons

\section{Recommended Citation}

Schiff G, Seoane-Vazquez E, Wright A. Incorporating indications into medication ordering: Time to enter the age of reason. $N$ Engl J Med. 2016;375(4):306-309. doi: 10.1056/NEJMp1603964

This Article is brought to you for free and open access by the School of Pharmacy at Chapman University Digital Commons. It has been accepted for inclusion in Pharmacy Faculty Articles and Research by an authorized administrator of Chapman University Digital Commons. For more information, please contact laughtin@chapman.edu. 


\section{Incorporating Indications into Medication Ordering — Time to Enter the Age of Reason}

\section{Comments}

This article was originally published in New England Journal of Medicine, volume 375, issue 4, in 2016. DOI: 10.1056/NEJMp1603964

\section{Copyright}

Massachusetts Medical Society 


\title{
Incorporating Indications into Medication Ordering - Time to Enter the Age of Reason
}

\author{
Gordon D. Schiff, M.D., Enrique Seoane-Vazquez, Ph.D., and Adam Wright, Ph.D.
}

\begin{abstract}
A 1833 article in the Boston A Medical and Surgical Journal (forerunner of the New England Journal of Medicine) explained why prescriptions should be written in Latin to protect patients from knowledge of the names of and indications for the prescribed drugs:
\end{abstract}

"The question is often asked, why physicians do not write . . . prescriptions in English. The answer is obvious - that if they did, the patient would often be less benefited than he now is. There are very few minds which have sufficient firmness, during the continuance of disease, to reason calmly on the probable effects of remedies, and to compare their wonted action ... with the indication to be fulfilled in the particular case. . . . The only state in which the mind can rest ... during severe illness, is that of implicit reliance in the skill of the physician, and an entire acquiescence in the course adopted, without the slightest question or argument."1

In our current era of transparent, patient-centered medicine, such sentiments would hardly be accepted. Unfortunately, patients are often still in the dark regarding the purposes of their medications. It is said that there are five "rights" required for safe medication ordering and use: the right patient, the right drug, the right dose, the right time, and the right route. But there's a sixth element that must be correct - and we believe it's time to add to each prescription an ingredient that's currently conspicuously missing: the right indication. This pivotal element affects and complements the other five, and considering it a sixth "right" would inform and enhance the safety of each prescription. With most prescriptions now being written electronically, this addition is particularly timely, since electronic medication ordering provides the vehicle for incorporating the indication into prescribing - and is handicapped in various ways without it.

Indications-based prescribing can contribute to better prescribing and medication use in multiple, synergistic ways (see table). First, when medication choices are narrowed to those indicated for a specific problem, decisions are much less prone to error. Staff and patients will be able to more easily recognize any mismatches and intercept prescribing or dispensing errors. Properly designed ordering systems could, for example, prevent common errors related to drugs whose names look and sound similar, such as inadvertent ordering of hydralazine for itching or hydroxyzine for hypertension. An indications-based computer prescribing system could render such drugindication mismatches impossible or at least make them easier for pharmacists to detect.

Second, we know that if patients are to understand and adhere to their medication regimens, they need to know the reason each medication is being prescribed. Having this knowledge has been shown to be associated with better adherence and fewer errors, ${ }^{2}$ yet patients often do not know the indications for some or all of their medications. ${ }^{3}$ Pharmacists, visiting nurses, and caregiving relatives also need this information, but they are often even more in the dark about the reason for a given prescription. Presented with a choice, most patients prefer instructional leaflets and prescription labels that include indications to those that don't include indications. ${ }^{4}$ Knowledge of the indication can also empower patients to question the necessity of a medication.

Third, prescribers need and want help choosing the best drugs for their patients' problems. Busy clinicians may not have time to look up recommended choices whenever they encounter problems beyond the limited repertoire they can hold in their heads. How many physicians can keep up with and recall the current regimen for Helicobacter pylori or gonorrhea infection? With pharmacotherapy choices and regimens becoming increasingly complex, there's a need for efficient, trustworthy, timely support for prescribing decisions. Since each prescription logically starts with a clinical problem or diagnosis, we should make it easy for prescribers to enter the indication (or click on a problem from the problem list) and have the system present the best thera- 
Potential Benefits

Improve medication safety

Help recognize and prevent wrong-patient medication errors

Identify, intercept, or avoid wrong-drug errors (e.g., errors due to pull-down menu or name confusion)

Ensure proper dosing regimen for particular condition

Better educate and empower patients about medications

Allow patients to understand and keep their various medications straight

Facilitate patient adherence by improving understanding

Enhance shared decision making and facilitate patients' asking questions about their medications

Improve health care team communication (physicians, pharmacists, patients, family, home care nurses)

Recognize roles and importance of entire team and teamwork in managing medications

Reduce need for pharmacist interventions and calls to and from pharmacy

Aid care transitions by communicating reasons for medications

Provide information to help counsel patients or help them take their medications

Facilitate medication reconciliation

Organize medication list by indication to help all participants reconcile therapy

Knowing reason why medication was started permits evaluation of whether safe to discontinue

Increase speed and efficiency of prescribing by presenting drug choices for that indication

Improve documentation of health problem list

Allow integration of problem list with medications prescribed

Support reorganizing medication list for more logical grouping

Improve appropriate use of medications by prescribers

Support selection of right and targeted medication choices

Reduce use of "never-indicated" drugs

Identify, support, learn from off-label use

Facilitate reimbursement coding, streamline prior authorization process

Support quality, outcomes, and effectiveness research

To evaluate outcomes need to know reason medication given

\section{Challenges}

Concerns about potential for extra prescriber time and effort

Added burden with paper prescriptions, requirements to add diagnosis to laboratory orders

Patient privacy concerns

Limited evidence base to support change

No randomized trials; only limited data showing that selected use of indications has been beneficial

Complexities in defining and creating indications

Empirical treatment when no definite diagnosis exists

Which terminologies to use (symptom, health problem, diagnosis, ICD-10, SNOMED-CT)

Complexities in differentiating billing diagnosis codes for reimbursement versus drug indication

Standardizing and maintaining indications knowledge databases

Drugs being given for multiple indications (e.g., ACE inhibitor for both congestive heart failure and blood pressure control)

Complexities in creating "smart" drug recommendations based on indications

Need to incorporate patient allergies, contraindicated coexisting conditions and laboratory values

Ensuring choices that avoid current medications and drugs previously used that have failed

Insurance and formulary requirements need to be incorporated and are often complex

Competing options for alternative ways to capture or infer indications

Complexities in transmitting indication information from $\mathrm{CPOE}$ to pharmacy, then to patient

Interoperability between EHRs and pharmacy systems

Medications are typically prescribed without structured record of indication in the EHR

Limited real estate for placing indication on prescription bottle labels

Legal and billing issues

Potential for inhibiting legitimate off-label use, reimbursement

Clinical autonomy concerns

Overcoming policy and market fragmentation (EHR/knowledge vendor/PBM/payer differing indications)

* Collated from the Agency for Healthcare Research and Quality-Brigham and Women's Hospital Indications-Based Prescribing webinars held April 2015 to June 2016. ${ }^{5}$ ACE denotes angiotensin-converting enzyme, CPOE computerized physician order entry, EHR electronic health record, ICD-10 International Classification of Diseases, 10th revision, PBM pharmacy benefit manager, and SNOMED-CT Systematized Nomenclature of Medicine - Clinical Terms.

peutic alternatives. This approach would not only enable the automatic capturing and recording of the indication, but also streamline prescribing with suggestions of the most appropriate choices.

Ideally, such a system would be "smart" enough to base suggestions on data in the electronic medical record, including the patient's allergies (which could trigger suggestions for appropriate alternatives), prior and current medications (so that the prescriber could avoid adverse interactions or avoid unknowingly repeating drugs that had already been tried unsuccessfully), relevant contra- indications, and insurance and formulary requirements. It's important that such recommendations be designed to provide sufficient flexibility to maintain prescribers' choice and autonomy.

Fourth, knowledge of indications is key to getting prescribers, pharmacists, nurses, and pa- 
tients on the same page regarding what is being treated and what outcomes are desired. Pharmacists who are uncertain about the reason a medication is being given will be unable to explain it to the patient. For decades, pharmacists' organizations have advocated for including indications on prescriptions, but physicians have objected that it would take them too much time. Confidentiality is another legitimate concern. However, better communication within the health care team broadly conceived is not incompatible with protecting patient confidentiality, and protections provided by the Health is infinitely harder. Once all medications for hypertension or asthma are grouped together, duplicates that may need to be discontinued are easier to spot. Discontinuing unneeded medications is an important related task, but when one doesn't know why a medication was prescribed, guessing the indication and whether it's OK to discontinue it becomes risky.

Finally, one cannot meaningfully measure a drug's long-term effectiveness (or comparative effectiveness) without reference to the reason for its use. Including indications in prescriptions would permit clearer assessments and

\section{Knowledge of indications is key to getting prescribers, pharmacists, nurses, and patients on the same page regarding what is being treated and what outcomes are desired.}

Insurance Portability and Accountability Act should allay this concern. For special cases (for instance, mental health or HIV infection), systems could be designed to permit prescribers or patients to easily opt out of having the indication included on the label.

Fifth, problems with medication reconciliation are a substantial source of confusion and error, including inadvertent duplicate therapy and inappropriate continuation of medications that are no longer needed. Reconciling medications is now widely mandated, but when medications are listed randomly, alphabetically, or chronologically (as they generally are) rather than being organized by indication, the task comparisons. Similarly, efforts to oversee and learn from patterns in prescribing practices and adherence require information about indications in order to assess appropriateness, exceptions, and off-label prescribing practices. We envision a system in which an off-label indication could easily be selected for a medication being prescribed, but in which such prescriptions could then be tracked and evaluated to inform possible labeling revisions or prescribing improvements.

Because incorporating indications into prescriptions represents such a compelling opportunity for improving the safety and quality of prescribing, the Agency for Healthcare Research and Quality has funded a 3-year project that we are spearheading that has brought together key stakeholders for seven international Web conferences to clarify the rationale behind this paradigm, the challenges to implementing it, and ways to move it forward. ${ }^{5}$

Key to realizing universal indications-based prescribing will be designing a system that fits into and enhances workflow and leverages other information technology. Rather than burdening prescribers with adding indications to prescriptions, we are working with human-factors and usability engineers, information technology design specialists, and policy leaders to build a prototype that will allow prescribers to start from the indication or the patient's problem and will guide them toward the best choices. We will be testing our hypothesis that this system will result in a safer, more efficient way of ordering medications. We hope and expect that it can bring prescribing into the age of reason, benefiting patients, prescribers, pharmacists, and other members of the health care team. Indications are the link connecting a patient with a given drug; we believe that electronic prescribing needs to incorporate this missing link.

Disclosure forms provided by the authors are available at NEJM.org.

From the Division of General Internal Medicine, Brigham and Women's Hospital (G.D.S., A.W.), Harvard Medical School (G.D.S., A.W.), and the Massachusetts College of Pharmacy and Health Sciences (E.S.-V.) - all in Boston.

1. Latin prescriptions. Boston Med Surg J 1833;9:98-9.

2. Kuntz JL, Safford MM, Singh JA, et al. Patient-centered interventions to improve medication management and adherence: 
a qualitative review of research findings. Patient Educ Couns 2014;97:310-26.

3. Persell SD, Heiman HL, Weingart SN, et al. Understanding of drug indications by ambulatory care patients. Am J Health Syst Pharm 2004;61:2523-7.

4. Zargarzadeh AH, Law AV. Design and test of preference for a new prescription medication label. Int J Clin Pharm 2011;33: 252-9.

5. Brigham Project Team on AHRQ Enhancing Medication Safety CPOE Safety and Quality by Indications Based Prescribing. Improving HIT prescribing safety by incorporating indications into computerized prescriber order entry (CPOE) (Webi- nar Series 2015-2016). AHRQ Center for Education on Therapeutics. CHAIN Online. 2015 (http://www.chainonline.org/research -tools/improving-hit-prescribing-safety).

DOI: 10.1056/NEJMp1603964

Copyright $\odot 2016$ Massachusetts Medical Society. 